\title{
CRADA BNL-C-95-07
}

I. ABSTRACT

The number of bases that can be read in a single run by a DNA sequencing instrument that detects fluorophore labeled DNA arriving at a "finish-line" located a fixed distance from the starting wells is influenced by numerous parameters. Strategies for improving the length-of-read of a DNA sequencer can be based on quantitative models of the separation of DNA by gel electrophoresis. The dispersion function of the electrophoretic system - the relationship between molecular contour length and time of arrival at the detector - is useful in characterizing the performance of a DNA sequencer. We adapted analytical representations of dispersion functions, originally developed for snapshot imaging of DNA gels, (samples electrophoresed for constant time), to finish-line imaging, and demonstrated that a logistic-type function with non-integral exponent is required to describe the experimental data. We use this dispersion function to determine the resolution length and resolving power of a LI-COR DNA sequencing system and a custom built capillary gel electrophoresis system, and discuss the factors that presently limit the number of bases that can be determined reliably in a single sequencing run.

Above, we described a quantitative measure of electrophoretic resolution called Resolving Power that can be computed for individual bands of DNA (or other molecules) separated by electrophoresis. An alternate approach is to determine analytical functions, based on a few experimentally determined parameters, that describe the resolving power of a particular electrophoretic system for all of the length classes of molecules that are separated. Such analytical functions have been obtained for single-stranded DNA separated in a polyacrylamide gel and detected at a fixed distance from the origin of electrophoresis. Six experimentally determined constants are required to describe the analytical function for resolving power in this system: four constants describe the mobility of DNA in the gel as a function of molecular length (or time of arrival at the detector), and two describe the width of the bands as a function of the length of molecular length (or time of arrival at the detector).

One of our goals is to develop systematic methods of improving the resolving power of longer molecules, and hence extending the number of bases that can be determined in a single sequencing experiment. Our approach is to determine how the small number $(\approx 6)$ of parameters that describe resolving power depend on experimentally controllably conditions (e.g. gel composition, electric field strength, temperature ...). Knowing such relationships should permit systematic selection of combinations of experimental conditions that improve resolving power. We show the relationship between the four parameters describing DNA mobility as a function of molecular length (mass) for systematic variations of gel composition and electric field strength. The data set employed is for double-stranded DNA separated in agarose gels, but the principles are similar to those encountered in DNA sequencing studies.

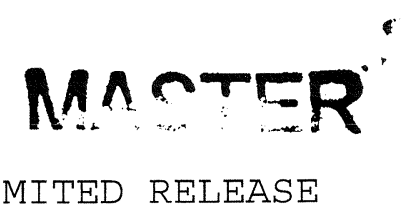




\section{REPORT}

\section{A. Accomplishments}

1. Theory of Resolving Power and its Relation to the Performance of a Automated DNA Sequencer

We developed the theory of electrophoretic resolving power to include both snap-shot imaging (as used with conventional gels) and finish-line imaging (as used in automated DNA sequencers). ${ }^{1}$

\section{Analytical Functions for Dispersion Functions}

Experimental data developed with a LI-COR sequencer demonstrated conclusively that the hyperbolic form of the electrophoretic dispersion function is qualitatively inadequate to represent the experimental data. We showed, however, that logistic dispersion functions provide a mush better representation of experimental data. ${ }^{1}$

\section{Analytical Descriptions of Resolving Power}

We developed complete descriptions of resolving power in terms of just 6 experimentally measurable variables. We were able to solve to obtain the location of the peak of the resolving power, and developed an new, ad hoc, measure of the actual experimental limit or resolution that agreed with empirical results obtained by LI-COR.

\section{Relating Experimental Parameters with the Analytical Theory.}

We showed for the case of double stranded DNA migrating through agarose gels that the four parameters of the gel dispersion function can be linked to experimentally controllable parameters such as electric field strength and gel concentration. 2

\section{B. Problems}

Pulsed field electrophoresis was shown not to increase length of read.

\section{Industry Benefits}

LI-COR received increased insight into the performance of their instrument and potential paths for increasing the "length of sequence read" and other paths to increased performance.

\section{Laboratory Benefits}

The laboratory has already published two research manuscripts describing the mechanisms of determining resolution in gel electrophoresis and in improving the analytical forms of dispersion functions involved in gel electrophoresis. Such data will benefit studies in which gel electrophoresis is used to quantify the damage produced in DNA by ultraviolet and ionizing radiation, and hence advance ongoing research programs at the laboratory. 


\section{E. Potential Benefits from pursuing follow-on work}

The program terminated just as we demonstrated how experimentally controllable parameters such as gel concentration and electric field strength can be related to the dispersion function. To determine how such experimentally controllable parameters actually effect electrophoretic resolving power, we need corresponding data on band widths. Such data would then make possible predictions of the optimum experimental parameters to use to obtain longer lengths of read of DNA sequences.

\section{REFERENCES}

${ }^{1}$ J. C. Sutherland, K. J. Reynolds, and D. J. Fisk, "Dispersion functions and factors that determine resolution for DNA sequencing by gel electrophoresis," Proceedings of the Society for Photo-Optical Instrumentation Engineers 2680, 326-340, 1996.

2 J. C. Sutherland, "Linking electrophoretic resolution with experimental conditions," Proceedings of the Society for Photo-Optical Instrumentation Engineers 2985, 47-60, 1997. 